BULLETIN OF THE

AMERICAN MATHEMATICAL SOCIETY

Volume 77, Number 6, November 1971

\title{
A STRUCTURE THEOREM FOR COMPLETE NONCOMPACT HYPERSURFACES OF NONNEGATIVE CURVATURE
}

\author{
BY H. WU ${ }^{1}$
}

Communicated by I. Singer, May 20, 1971

The convexity theorem of Sacksteder-van Heijenoort [4] states that if $M$ is a $C^{\infty} n$-dimensional $(n>1)$ complete orientable Riemannian manifold of nonnegative sectional curvature with positive curvature at one point, then every isometric immersion $x: M \rightarrow R^{n+1}$ is an imbedding and $x(M)$ bounds an open convex subset of $R^{n+1}$; furthermore $M$ is diffeomorphic to either $R^{n}$ or $S^{n}$ (unit $n$-sphere). The purpose of this note is to announce a structure theorem that complements the above result of Sacksteder and van Heijenoort. Full details will appear in a forthcoming monograph on convexity and rigidity of hypersurfaces.

TheOREM. Let $M$ be a $C^{\infty}$ hypersurface in $R^{n+1}(n>1)$ which is complete, noncompact, orientable with nonnegative sectional curvature, which is in addition all positive at one point, then:

(1) The spherical image of $M$ in the unit sphere $S^{n}$ has a geodesically convex closure, which lies in a closed hemisphere.

(2) The total curvature of $M$ (cf. Chern-Lashof [2]) does not exceed one.

(3) $M$ is a pseudograph (see below for definition) over one of its tangent planes.

(4) $M$ has infinite volume.

Corollary. Suppose the sectional curvature of $M$ is in fact everywhere positive, then:

(5) The spherical map is a diffeomorphism onto a geodesically convex open subset of $S^{n}$. Consequently the spherical image lies in an open hemisphere.

(6) Coordinates in $R^{n+1}$ can be so chosen that $M$ is tangent to the hyperplane $x_{n+1}=0$ at the origin, and there is a nonnegative strictly convex function (i.e. its Hessian is everywhere positive definite) $f\left(x_{1}, \cdots, x_{n}\right)$ defined in a convex domain of $\left\{x_{n+1}=0\right\}$ such that $M$ is exactly the graph of $f$.

Remarks. (A) A $C^{\infty}$ convex hypersurface $M$ (i.e. $M$ is the full boundary of an open convex set) in $R^{n+1}$ is said to form a pseudograph

AMS 1969 subject classifications. Primary 5374; Secondary 5375.

1 Sloan Fellow. Also partially supported by the National Science Foundation. 
over the tangent plane $H$ if and only if:

(a) $M$ lies above $H$, i.e. designating a closed half-space of $H$ as being above $H$, we have that $M$ lies in this half-space.

(b) Let $\pi: R^{n+1} \rightarrow H$ be the orthogonal projection and let $A=\pi(M)$. Then over the interior $A^{\circ}$ (of $A$ as a subset of $H$ ), $M$ is the graph of a $C^{\infty}$ function.

(c) For every $a \in A-A^{\circ}, M \cap \pi^{-1}(a)$ is a closed semi-infinite straight line segment.

(d) Every hyperplane strictly above $H$ intersects $M$ at a diffeomorph of the unit $(n-1)$-sphere $S^{n-1}$.

(B) When $n=2$ and the curvature of $M$ is everywhere positive, this theorem (as well as the theorem of Sacksteder-van Heijenoort) was first proved by Stoker [5].

(C) Assertions (2)-(6) above all follow from assertion (1). We actually prove a more general result than (1):

Proposition. Let $C$ be an open convex subset of $R^{n+1}(n \geqq 1)$ with connected boundary $M$ and let $\gamma: M \rightarrow S^{n}$ be the spherical map (in the sense of Alexandrov, see Busemann [1]). Then the closure of $\gamma(M)$ is geodesically convex.

The proof of this Proposition is achieved quite simply by employing the concept of the barrier cone of a convex set. See Rockafellar [3].

(D) Neither (1) nor the Proposition is true if the word "closure" is deleted. (Cf. Busemann [1, p. 25, (4.4)].)

(E) The Proposition has applications in the theory of convex surfaces, e.g. Alexandrov's theory of spherical measures on an open convex surface (Busemann [1, p. 31]) or the rigidity and nonrigidity theorems of Pogorelov and Olovyanishnikov on open convex surfaces (Busemann [1, pp. 167-168]).

\section{BIBLIOGRAPHY}

1. H. Busemann, Convex surfaces, Interscience Tracts in Pure and Appl. Math., no. 6, Interscience, New York, 1958. MR 21 \#3900.

2. S. S. Chern and R. K. Lashof, On the total curvature of immersed manifolds. II, Michigan Math. J. 5 (1958), 5-12. MR 20 \#4301. 1970.

3. R. T. Rockafellar, Convex analysis, Princeton Univ. Press, Princeton, N.J.,

4. R. Sacksteder, On hypersurfaces with nonnegative sectional curvatures, Amer. J. Math. 82 (1960), 609-630. MR 22 \#7087.

5. J. J. Stoker, Über die Gestalt der positiv gekrümmten offenen Flächer, Compositio Math. 3 (1936), 55-88.

University of California, Berkeley, California 94720

Princeton University, Princeton, New Jersey 08540 